\title{
Fixed points of mappings satisfying contractive condition of integral type in modular spaces endowed with a graph
}

\author{
Mahpeyker Öztürk*, Mujahid Abbas ${ }^{2,3}$ and Ekber Girgin
}

*Correspondence:
mahpeykero@sakarya.edu.tr
${ }^{1}$ Department of Mathematics,
Sakarya University, Sakarya, 54187,
Turkey
Full list of author information is
available at the end of the article

\begin{abstract}
Jachymski (Proc. Am. Math. Soc. 136:1359-1373, 2008) gave a modified version of a Banach fixed point theorem on a metric space endowed with a graph. The aim of this paper is to present fixed point results of mappings satisfying integral type contractive conditions in the framework of modular spaces endowed with a graph. Some examples are presented to support the results proved herein. Our results generalize and extend various comparable results in the existing literature.
\end{abstract}

MSC: $47 \mathrm{H} 10 ; 54 \mathrm{H} 25 ; 54 \mathrm{E} 50$

Keywords: connected graph; modular space; Banach G-contraction; integral type contraction

\section{Introduction}

Fixed point theory for nonlinear mappings is an important subject of nonlinear functional analysis. One of the basic and the most widely applied fixed point theorem in all of analysis is the 'Banach (or Banach-Caccioppoli) contraction principle' due to Banach [1]. This Banach contraction principle [1] is a simple and powerful result with a wide range of applications, including iterative methods for solving linear, nonlinear, differential, integral, and difference equations. Due to its applications in mathematics and other related disciplines, the Banach contraction principle has been generalized in many directions.

The existence of fixed points in ordered metric spaces has been discussed by Ran and Reurings [2]. Recently, many researchers have obtained fixed point and common fixed point results for single valued maps defined on partially ordered metric spaces (see, e.g., $[3,4])$. Jachymski [5] investigated a new approach in metric fixed point theory by replacing an order structure with graph structure on a metric space. In this way, the results proved in ordered metric spaces are generalized (see for details [5] and the references therein). For further work in this direction, we refer to, e.g., [6-8].

In 1968, Kannan [9] proved a fixed point theorem for a map satisfying a contractive condition that did not require continuity at each point. This paper led to the genesis for a multitude of fixed point papers over the next two decades. Since then, there have been many theorems dealing with mappings satisfying various types of contractive inequalities involving linear and nonlinear expressions. For a thorough survey, we refer to [10] and the references therein. On the other hand, Branciari [11] obtained a fixed point theorem 
for a single valued mapping satisfying an analog of Banach's contraction principle for an integral type inequality. Recently, Akram et al. [12] introduced a new class of contraction maps, called $A$-contractions, which is a proper generalization of Kannan's mappings [9], Bianchini's mappings [13], and Reich type mappings [14].

The theory of modular spaces was initiated by Nakano [15] in connection with the theory of ordered spaces which was further generalized by Musielak and Orlicz [16] (see also [17-21]). The study of fixed point theory in the context of modular function spaces was initiated by Khamsi et al. [22] (see also [22-26]). Also, some fixed point theorems have been proved for mappings satisfying contractive conditions of integral type in modular space $[27,28]$.

In this paper, we introduce three new classes of mappings satisfying integral type contractive conditions in the setup of modular space endowed with graphs. We study the existence, uniqueness, and iterative approximations of fixed points for such mappings. Our results extend, unify, and generalize the comparable results in $[5,11,12]$.

\section{Preliminaries}

A mapping $T$ from a metric space $(X, d)$ into $(X, d)$ is called a Picard operator (PO) if $T$ has a unique fixed point $z \in X$ and $\lim _{n \rightarrow \infty} T^{n} x=z$ for all $x \in X$.

Define $\Phi=\left\{\varphi: \mathbb{R}_{+} \rightarrow \mathbb{R}_{+}: \varphi\right.$ is a Lebesgue integral mapping which is summable, nonnegative and satisfies $\int_{0}^{\epsilon} \varphi(t) d t>0$, for each $\left.\epsilon>0\right\}$.

Let $A=\left\{\alpha: \mathbb{R}_{+}^{3} \rightarrow \mathbb{R}_{+}: \alpha\right.$ is continuous and $a \leq k b$ for some $k \in[0,1)$ whenever $a \leq$ $\alpha(a, b, b)$ or $a \leq \alpha(b, a, b)$ or $a \leq \alpha(b, b, a)$ for all $a, b\}$.

Let $\psi: \mathbb{R}_{+} \rightarrow \mathbb{R}_{+}$be a nondecreasing mapping which satisfies the following conditions:

$\left(\psi_{1}\right) \psi(x)=0$ if and only if $x=0$;

$\left(\psi_{2}\right)$ for a sequence $\left\{x_{n}\right\}$ in $\mathbb{R}_{+}$, we have $\psi\left(x_{n}\right) \rightarrow 0$ if and only if $x_{n} \rightarrow 0$ as $n \rightarrow \infty$;

$\left(\psi_{3}\right)$ for every $x, y \in \mathbb{R}_{+}$, we have $\psi(x+y) \leq \psi(x)+\psi(y)$.

The collection of all such mappings will be denoted by $\Psi$.

Define

$$
\Phi_{1}=\left\{\phi: \mathbb{R}_{+} \rightarrow \mathbb{R}_{+}: \phi \text { is increasing, upper semi-continuous and } \phi(t)<t, \forall t>0\right\} .
$$

Theorem $2.1[11]$ Let $(X, d)$ be a complete metric space, $\eta \in[0,1)$, and $T: X \rightarrow X$ a mapping. Suppose that

$$
\int_{0}^{d(T x, T y)} \varphi(t) d t \leq \eta \int_{0}^{d(x, y)} \varphi(t) d t
$$

is satisfied for every $x, y \in X$, where $\varphi \in \Phi$. Then $T$ has a unique fixed point $z \in X$ and for each $x \in X$, we have $\lim _{n \rightarrow \infty} T^{n} x=z$.

Lemma 2.2 [29] Let $(X, d)$ be a metric space, $\varphi \in \Phi$, and $\left\{x_{n}\right\}$ a nonnegative sequence. Then

(a) $\lim _{n \rightarrow \infty} x_{n}=x$ implies that $\lim _{n \rightarrow \infty} \int_{0}^{x_{n}} \varphi(t) d t=\int_{0}^{x} \varphi(t) d t$;

(b) $\lim _{n \rightarrow \infty} \int_{0}^{x_{n}} \varphi(t) d t=0$ if and only if $\lim _{n \rightarrow \infty} x_{n}=0$. 
Definition 2.3 [12] A selfmap $T$ on a metric space $X$ is called an $A$-contraction if for any $x, y \in X$ and for some $\alpha \in A$, the following condition holds:

$$
d(T x, T y) \leq \alpha(d(x, y), d(x, T x), d(y, T y)) .
$$

Now, we recall some basic facts and notations as regards modular spaces. For more details the reader may consult [16].

Definition 2.4 Let $X$ be an arbitrary vector space. A functional $\rho: X \rightarrow[0, \infty]$ is called modular if for any arbitrary $x, y$ in $X$ :

$\left(\mathrm{m}_{1}\right) \rho(x)=0$ if and only if $x=0$;

$\left(\mathrm{m}_{2}\right) \rho(\alpha x)=\rho(x)$ for every scalar $\alpha$ with $|\alpha|=1$;

$\left(\mathrm{m}_{3}\right) \rho(\alpha x+\beta y) \leq \rho(x)+\rho(y)$ if $\alpha+\beta=1, \alpha \geq 0, \beta \geq 0$.

If $\left(\mathrm{m}_{3}\right)$ is replaced by $\rho(\alpha x+\beta y) \leq \alpha^{s} \rho(x)+\beta^{s} \rho(y)$ if $\alpha^{s}+\beta^{s}=1$, where $s \in(0,1], \alpha \geq 0$, $\beta \geq 0$ then we say that $\rho$ is $s$-convex modular. If $s=1$, then $\rho$ is called convex modular.

$\rho: R \rightarrow[0, \infty]$ defined by $\rho(x)=\sqrt{|x|}$ is a simple example of a modular functional.

The vector space $X_{\rho}$ given by

$$
X_{\rho}=\{x \in X ; \rho(\lambda x) \rightarrow 0 \text { as } \lambda \rightarrow 0\}
$$

is called a modular space. In general the modular $\rho$ is not sub-additive and therefore does not behave as a norm or a distance. One can associate to a modular an $x$-norm.

Remark 2.5 [28] The following are immediate consequences of condition $\left(\mathrm{m}_{3}\right)$ :

$\left(\mathrm{r}_{1}\right)$ if $a, b \in \mathbb{R}$ (set of all real numbers) with $|a|<|b|$, then $\rho(a x)<\rho(b x)$ for all $x \in X$;

$\left(\mathrm{r}_{2}\right)$ if $a_{1}, \ldots, a_{n}$ are nonnegative real numbers with $\sum_{i=1}^{n} a_{i}=1$, then we have

$$
\rho\left(\sum_{i=1}^{n} a_{i} x_{i}\right) \leq \sum_{i=1}^{n} \rho\left(x_{i}\right) \quad\left(x_{1}, \ldots, x_{n} \in X\right) .
$$

Define the $\rho$-ball, $B_{\rho}(x, r)$, centered at $x \in X_{\rho}$ with radius $r$ as

$$
B_{\rho}(x, r)=\{h \in X \rho ; \rho(x-h) \leq r\} .
$$

A point $x \in X_{\rho}$ is called a fixed point of $T: X_{\rho} \rightarrow X_{\rho}$ if $T(x)=x$.

A function modular is said to satisfy the $\Delta_{2}$-type condition if there exists $K>0$ such that for any $x \in X_{\rho}$ we have $\rho(2 x) \leq K \rho(x)$. A modular $\rho$ is said to satisfy the $\Delta_{2}$-condition if $\rho\left(2 x_{n}\right) \rightarrow 0$ as $n \rightarrow \infty$, whenever $\rho\left(x_{n}\right) \rightarrow 0$ as $n \rightarrow \infty$.

Definition 2.6 Let $X_{\rho}$ be a modular space. The sequence $\left\{x_{n}\right\} \subset X_{\rho}$ is said to be:

$\left(\mathrm{t}_{1}\right) \rho$-convergent to $x \in X_{\rho}$ if $\rho\left(x_{n}-x\right) \rightarrow 0$ as $n \rightarrow \infty$;

$\left(\mathrm{t}_{2}\right) \rho$-Cauchy if $\rho\left(x_{n}-x_{m}\right) \rightarrow 0$ as $n$ and $m \rightarrow \infty$.

$X_{\rho}$ is $\rho$-complete if any $\rho$-Cauchy sequence is $\rho$-convergent. Note that $\rho$-convergence does not imply $\rho$-Cauchy since $\rho$ does not satisfy the triangle inequality. In fact, one can show that this will happen if and only if $\rho$ satisfies the $\Delta_{2}$-condition. 
Proposition 2.7 [30] Let $X_{\rho}$ be a modular space. If $a, b \in \mathbb{R}_{+}$with $b \geq a$, then $\rho(a x) \leq$ $\rho(b x)$.

Proposition 2.8 [30] Suppose that $X_{\rho}$ is a modular space, $\rho$ satisfies the $\Delta_{2}$-condition and $\left\{x_{n}\right\}_{n \in \mathbb{N}}$ is a sequence in $X_{\rho}$. If $\rho\left(c\left(x_{n}-x_{n-1}\right)\right) \rightarrow 0$, then $\rho\left(\alpha l\left(x_{n}-x_{n-1}\right)\right) \rightarrow 0$, as $n \rightarrow \infty$, where $c, l, \alpha \in \mathbb{R}_{+}$with $c>l$ and $\frac{l}{c}+\frac{1}{\alpha}=1$. Now we give some basic definitions from graph theory needed in the sequel.

Throughout this paper, $\Delta=\{(x, x): x \in X\}$ denotes the diagonal of $X \times X$, where $X$ is any nonempty set. Let $G$ be a directed graph such that the set $V(G)$ of its vertices coincides with $X$ and $E(G)$ be the set of edges of the graph such that $\Delta \subseteq E(G)$. Further assume that $G$ has no parallel edge and $G$ is a weighted graph in the sense that each edge is assigned a distance $d(x, y)$ between their vertices $x$ and $y$ and each vertex $x$ is assigned a weight $d(x, x)$. The graph $G$ is identified by the pair $(V(G), E(G))$.

If $x$ and $y$ are vertices of $G$, then a path in $G$ from $x$ to $y$ of length $k \in N$ is a finite sequence $\left\{x_{n}\right\}, n \in\{0,1,2, \ldots, k\}$ of vertices such that $x=x_{0}, \ldots, x_{k}=y$ and $\left(x_{i-1}, x_{i}\right) \in E(G)$ for $i \in\{1,2, \ldots, k\}$.

Recall that a graph $G$ is connected if there is a path between any two vertices and it is weakly connected if $\tilde{G}$ is connected, where $\tilde{G}$ denotes the undirected graph obtained from $G$ by ignoring the direction of edges. Denote by $G^{-1}$ the graph obtained from $G$ by reversing the direction of the edges. Thus

$$
E\left(G^{-1}\right)=\{(x, y) \in X \times X:(y, x) \in E(G)\} .
$$

Since it is more convenient to treat $\tilde{G}$ as a directed graph for which the set of its edges is symmetric, under this convention we have

$$
E(\tilde{G})=E(G) \cup E\left(G^{-1}\right) .
$$

Let $G_{x}$ be the component of $G$ consisting of all the edges and vertices which are contained in some path in $G$ beginning at $x$. If $G$ is such that $E(G)$ is symmetric, then for $x \in V(G)$, the equivalence class $[x]_{G}$ defined on $V(G)$ by the rule $R(x R y$ if there is a path in $G$ from $x$ to $y$ ) is such that $V\left(G_{x}\right)=[x]_{G}$.

Definition 2.9 [5, Definition 2.1] A mapping $T: X \rightarrow X$ is called a Banach $G$-contraction if and only if:

(a) for each $x, y$ in $X$ with $(x, y) \in E(G)$, we have $(T(x), T(y)) \in E(G)$, that is, $T$ preserves edges of $G$;

(b) there exists $\alpha$ in $(0,1)$ such that for each $x, y \in X$ with $(x, y) \in E(G)$ implies

$$
d(T(x), T(y)) \leq \alpha d(x, y) .
$$

That is, $T$ decreases weights of edges of $G$.

For any $x, y \in V^{\prime},(x, y) \in E^{\prime}$ such that $V^{\prime} \subseteq V(G), E^{\prime} \subseteq E(G),\left(V^{\prime}, E^{\prime}\right)$ is called a subgraph of $G$.

\section{Main results}

In this section, we obtain several fixed point results in the setup of a modular space endowed with a graph. We start with the following definitions. Let $X_{\rho}$ be a modular space 
endowed with a graph $G$ and let $T: X_{\rho} \rightarrow X_{\rho}$ be a mapping. Denote

$$
X_{T}=\{x \in X:(x, T x) \in E(G)\} .
$$

Definition 3.1 Let $\left\{T^{n} x\right\}$ be a sequence, there exists $C>0$ such that

$$
\rho\left(C\left(T^{n} x-x^{*}\right)\right) \rightarrow 0 \quad \text { for } x^{*} \in X_{\rho}
$$

and

$$
\left(T^{n} x, T^{n+1} x\right) \in E(G) \quad \text { for all } n \in \mathbb{N} .
$$

Then a graph $G$ is called a $C_{\rho}$-graph if there exists a subsequence $\left\{T^{n_{p}} x\right\}$ of $\left\{T^{n} x\right\}$ such that $\left(T^{n_{p}} \mathcal{X}, x^{*}\right) \in E(G)$ for $p \in \mathbb{N}$.

Definition 3.2 A mapping $T: X_{\rho} \rightarrow X_{\rho}$ is called orbitally $G_{\rho}$-continuous for all $x, y \in X_{\rho}$ and any sequence $\left(n_{p}\right)_{p \in \mathbb{N}}$ of positive integers, if there exists $C>0$ such that

$$
\begin{aligned}
& \rho\left(C\left(T^{n_{p}} x-y\right)\right) \rightarrow 0, \quad\left(T^{n_{p}} x, T^{n_{p}+1} x\right) \in E(G) \text { imply } \\
& \rho\left(C\left(T\left(T^{n_{p}} x\right)-T(y)\right)\right) \rightarrow 0,
\end{aligned}
$$

as $p \rightarrow \infty$

Definition 3.3 A mapping $T$ is called a $(G, A)_{\rho}$-contraction if it satisfies the following conditions:

(A $\left.A_{1}\right) T$ preserves edges of $G$;

$\left(\mathrm{A}_{2}\right)$ there exist nonnegative numbers $l, c$ with $l<c$ such that

$$
\begin{aligned}
& \int_{0}^{\rho(c(T x-T y))} \varphi(t) d t \\
& \quad \leq \alpha\left(\int_{0}^{\rho(l(x-y))} \varphi(t) d t, \int_{0}^{\rho(l(x-T x))} \varphi(t) d t, \int_{0}^{\rho(l(y-T y))} \varphi(t) d t\right)
\end{aligned}
$$

holds for each $(x, y) \in E(G)$, and some $\alpha \in A$ and $\varphi \in \Phi$.

Remark 3.4 Let $T: X_{\rho} \rightarrow X_{\rho}$ be a $(G, A)_{\rho}$-contraction. If there exists $x_{0} \in X_{\rho}$ such that $T x_{0} \in\left[x_{0}\right]_{\tilde{G}}$, then

(i) $T$ is both a $\left(G^{-1}, A\right)_{\rho}$-contraction and a $(\tilde{G}, A)_{\rho}$-contraction,

(ii) $\left[x_{0}\right]_{\tilde{G}}$ is $T$-invariant and $\left.T\right|_{\left[x_{0}\right] \tilde{G}}$ is a $\left(\tilde{G}_{x_{0}}, A\right)_{\rho}$-contraction.

Lemma 3.5 Let $T: X_{\rho} \rightarrow X_{\rho}$ bea $(G, A)_{\rho}$-contraction. If $x \in X_{T}$, then there exists $r(x, T x) \geq$ 0 such that

$$
\int_{0}^{\rho\left(c\left(T^{n} x-T^{n+1} x\right)\right)} \varphi(t) d t \leq k^{n} r(x, T x)
$$

holds for all $n \in \mathbb{N}$, where $r(x, T x)=\int_{0}^{\rho(c(x-T x))} \varphi(t) d t$. 
Proof Let $x \in X_{T}$, that is, $(x, T x) \in E(G)$. Then by induction, we have $\left(T^{n} x, T^{n+1} x\right) \in E(G)$ for all $n \in \mathbb{N}$. Now, we have

$$
\begin{aligned}
& \int_{0}^{\rho\left(c\left(T^{n} x-T^{n+1} x\right)\right)} \varphi(t) d t \\
& \quad \leq \alpha\left(\int_{0}^{\rho\left(l\left(T^{n-1} x-T^{n} x\right)\right)} \varphi(t) d t, \int_{0}^{\rho\left(l\left(T^{n-1} x-T^{n} x\right)\right)} \varphi(t) d t, \int_{0}^{\rho\left(l\left(T^{n} x-T^{n+1} x\right)\right)} \varphi(t) d t\right) \\
& \quad \leq \alpha\left(\int_{0}^{\rho\left(l\left(T^{n-1} x-T^{n} x\right)\right)} \varphi(t) d t, \int_{0}^{\rho\left(l\left(T^{n-1} x-T^{n} x\right)\right)} \varphi(t) d t, \int_{0}^{\rho\left(c\left(T^{n} x-T^{n+1} x\right)\right)} \varphi(t) d t\right) .
\end{aligned}
$$

By the definition of $\alpha$, we obtain

$$
\int_{0}^{\rho\left(c\left(T^{n} x-T^{n+1} x\right)\right)} \varphi(t) d t \leq k \int_{0}^{\rho\left(l\left(T^{n-1} x-T^{n} x\right)\right)} \varphi(t) d t \leq k \int_{0}^{\rho\left(c\left(T^{n-1} x-T^{n} x\right)\right)} \varphi(t) d t
$$

for some $k \in(0,1)$. Thus we have

$$
\begin{aligned}
\int_{0}^{\rho\left(c\left(T^{n} x-T^{n+1} x\right)\right)} \varphi(t) d t & \leq k \int_{0}^{\rho\left(l\left(T^{n-1} x-T^{n} x\right)\right)} \varphi(t) d t \\
& \leq k \int_{0}^{\rho\left(c\left(T^{n-1} x-T^{n} x\right)\right)} \varphi(t) d t \\
& \leq k \cdot k \int_{0}^{\rho\left(l\left(T^{n-2} x-T^{n-1} x\right)\right)} \varphi(t) d t \\
& \leq k^{2} \int_{0}^{\rho\left(c\left(T^{n-2} x-T^{n-1} x\right)\right)} \varphi(t) d t \\
& \vdots \\
& \leq k^{n} \int_{0}^{\rho(l(x-T x))} \varphi(t) \\
& \leq k^{n} \int_{0}^{\rho(c(x-T x))} \varphi(t) .
\end{aligned}
$$

That is, $\int_{0}^{\rho\left(c\left(T^{n} x-T^{n-1} x\right)\right)} \varphi(t) d t \leq k^{n} r(x, T x)$ for all $n \in \mathbb{N}$, where $r(x, T x)=\int_{0}^{\rho(c(x-T x))} \varphi(t) d t$.

Theorem 3.6 Let $X_{\rho}$ be a $\rho$-complete modular space endowed with a graph $G$, where $\rho$ satisfies the $\Delta_{2}$-condition and let $T: X_{\rho} \rightarrow X_{\rho}$ be a $(\tilde{G}, A)_{\rho}$-contraction. If the set $X_{T}$ is nonempty, the graph $G$ is weakly connected and a $C_{\rho}$-graph, then $T$ is a PO.

Proof If $x \in X_{T}$, then $T x \in[x]_{\tilde{G}}$ and $\left(T^{n} x, T^{n+1} x\right) \in E(G)$ for all $n \in \mathbb{N}$. Let $m, n \in \mathbb{N}$ with $m>n$. Note that

$$
\begin{aligned}
& \rho\left(\frac{c}{m-n}\left(T^{n} x-T^{m} x\right)\right) \\
& \quad=\rho\left(\frac{c}{m-n}\left(T^{n} x-T^{n+1} x\right)+\frac{c}{m-n}\left(T^{n+1} x-T^{n+2} x\right)+\cdots+\frac{c}{m-n}\left(T^{m-1} x-T^{m} x\right)\right) \\
& \quad \leq \rho\left(c\left(T^{n} x-T^{n+1} x\right)\right)+\rho\left(c\left(T^{n+1} x-T^{n+2} x\right)\right)+\cdots+\rho\left(c\left(T^{m-1} x-T^{m} x\right)\right) .
\end{aligned}
$$


Using Lemma 3.5, we have

$$
\begin{aligned}
\int_{0}^{\rho\left(\frac{c}{m-n}\left(T^{n} x-T^{m} x\right)\right)} \varphi(t) d t \leq & \int_{0}^{\rho\left(c\left(T^{n} x-T^{n+1} x\right)\right)} \varphi(t) d t+\int_{0}^{\rho\left(c\left(T^{n+1} x-T^{n+2} x\right)\right)} \varphi(t) d t \\
& +\cdots+\int_{0}^{\rho\left(c\left(T^{m-1} x-T^{m} x\right)\right)} \varphi(t) d t \\
\leq & k^{n} r(x, T x)+k^{n+1} r(x, T x)+\cdots+k^{m-1} r(x, T x) \\
\leq & \frac{k^{n}}{1-k} r(x, T x) \rightarrow 0, \quad \text { as } n \rightarrow \infty .
\end{aligned}
$$

It follows that $\left\{\frac{c}{m-n} T^{n} x\right\}$ is a $\rho$-Cauchy sequence in $X_{\rho}$. Since $X_{\rho}$ is $\rho$-complete, there exists a point $x^{*} \in X_{\rho}$ such that $\rho\left(\frac{c}{m-n}\left(T^{n} x-x^{*}\right)\right) \rightarrow 0$. Consequently, $\rho\left(l\left(T^{n} x-x^{*}\right)\right) \rightarrow 0$.

Now we show that $x^{*}$ is a fixed point of $T$. As $\rho\left(\frac{c}{m-n}\left(T^{n} x-x^{*}\right)\right) \rightarrow 0,\left(T^{n} x, T^{n+1} x\right) \in E(G)$ for all $n \in \mathbb{N}$ and $G$ is a $C_{\rho}$-graph, there exists a subsequence $\left\{T^{n_{p}} x\right\}$ of $\left\{T^{n} x\right\}$ such that $\left(T^{n_{p}} x, x^{*}\right) \in E(G)$ for each $p \in \mathbb{N}$. Since $\left(T^{n_{p}} x, x^{*}\right) \in E(\tilde{G})$ and $T$ is a $(\tilde{G}, A)_{\rho}$-contraction, it follows that

$$
\begin{aligned}
& \int_{0}^{\rho\left(c\left(T^{n_{p}+1} x-T x^{*}\right)\right)} \varphi(t) d t \\
& \quad \leq \alpha\left(\int_{0}^{\rho\left(l\left(T^{n_{p}} x-x^{*}\right)\right)} \varphi(t) d t, \int_{0}^{\rho\left(l\left(T^{n_{p}} x-T^{n_{p}+1} x\right)\right)} \varphi(t) d t, \int_{0}^{\rho\left(l\left(x^{*}-T x^{*}\right)\right)} \varphi(t) d t\right),
\end{aligned}
$$

which on taking the limit as $p \rightarrow \infty$ gives

$$
\int_{0}^{\rho\left(c\left(x^{*}-T x^{*}\right)\right)} \varphi(t) d t \leq \alpha\left(0,0, \int_{0}^{\rho\left(l\left(x^{*}-T x^{*}\right)\right)} \varphi(t) d t\right) \leq \alpha\left(0,0, \int_{0}^{\rho\left(c\left(x^{*}-T x^{*}\right)\right)} \varphi(t) d t\right) .
$$

By the definition of function $\alpha$, we have

$$
\int_{0}^{\rho\left(c\left(x^{*}-T x^{*}\right)\right)} \varphi(t) d t \leq k \cdot 0=0 .
$$

From Lemma 2.2, it follows that $\rho\left(c\left(x^{*}-T x^{*}\right)\right)=0$ and $T x^{*}=x^{*}$.

Next, we prove that $x^{*}$ is a unique fixed point. Suppose that $T$ has another fixed point $y^{*} \in X_{\rho}-\left\{x^{*}\right\}$. Since $G$ is a $C_{\rho}$-graph, there exists a subsequence $\left\{T^{n_{p}} x\right\}$ of $\left\{T^{n} x\right\}$ such that $\left(T^{n_{p}} x, x^{*}\right) \in E(G)$ and $\left(T^{n_{p}} x, y^{*}\right) \in E(G)$ for each $p \in \mathbb{N}$. Furthermore, $G$ is weakly connected, $\left(x^{*}, y^{*}\right) \in E(\tilde{G})$, and we have

$$
\begin{aligned}
\int_{0}^{\rho\left(c\left(x^{*}-y^{*}\right)\right)} \varphi(t) d t & =\int_{0}^{\rho\left(c\left(T x^{*}-T y^{*}\right)\right)} \varphi(t) d t \\
& \leq \alpha\left(\int_{0}^{\rho\left(l\left(x^{*}-y^{*}\right)\right)} \varphi(t) d t, \int_{0}^{\rho\left(l\left(x^{*}-T x^{*}\right)\right)} \varphi(t) d t, \int_{0}^{\rho\left(l\left(y^{*}-T y^{*}\right)\right)} \varphi(t) d t\right) \\
& \leq \alpha\left(\int_{0}^{\rho\left(l\left(x^{*}-y^{*}\right)\right)} \varphi(t) d t, 0,0\right) \\
& \leq \alpha\left(\int_{0}^{\rho\left(c\left(x^{*}-y^{*}\right)\right)} \varphi(t) d t, 0,0\right)
\end{aligned}
$$


By the definition of $\alpha$ and Lemma 2.2, we have $\int_{0}^{\rho\left(c\left(x^{*}-y^{*}\right)\right)} \varphi(t) d t \leq k \cdot 0=0, \rho\left(c\left(x^{*}-y^{*}\right)\right)=$ 0 , and $x^{*}=y^{*}$.

In Theorem 3.6, if we replace the condition that $G$ is a $C_{\rho}$-graph with orbitally $G_{\rho}$-continuity of $T$, then we have the following theorem.

Theorem 3.7 Let $X_{\rho}$ be a $\rho$-complete modular space endowed with a graph $G$, where $\rho$ satisfies the $\Delta_{2}$-condition and let $T: X_{\rho} \rightarrow X_{\rho}$ be a $(\tilde{G}, A)_{\rho}$-contraction and orbitally $G_{\rho}$ continuous. If the set $X_{T}$ is nonempty and the graph $G$ is weakly connected, then $T$ is a PO.

Proof If $x \in X_{T}$, then Theorem 3.6 implies that $\left\{\frac{c}{m-n} T^{n} x\right\}$ is a $\rho$-Cauchy sequence in $X_{\rho}$. Owing to $\rho$-completeness of $X_{\rho}$, there exists $x^{*} \in X_{\rho}$ such that $\rho\left(\frac{c}{m-n}\left(T^{n} x-\right.\right.$ $\left.\left.x^{*}\right)\right) \rightarrow 0$. As $\left(T^{n} x, T^{n+1} x\right) \in E(G)$ for all $n \in \mathbb{N}$ and $T$ is orbitally $G_{\rho}$-continuous, we have $\rho\left(\frac{c}{m-n}\left(T\left(T^{n} x\right)-T\left(x^{*}\right)\right)\right) \rightarrow 0$, as $n \rightarrow \infty$. That is, $T x^{*}=x^{*}$. Assume that $y^{*}$ is another fixed point of $T$. Following arguments similar to those in the proof of Theorem 3.6, we obtain $y^{*}=x^{*}$.

Corollary 3.8 Let $X_{\rho}$ be a $\rho$-complete modular space endowed with a graph $G$, where $\rho$ satisfies the $\Delta_{2}$-condition and let $T: X_{\rho} \rightarrow X_{\rho}$ be edge-preserving, the set $X_{T}$ nonempty and graph $G$ be weakly connected and $a C_{\rho}$-graph. If there exist nonnegative numbers $l, c$ with $l<c$ such that

$$
\rho(c(T x-T y)) \leq \alpha(\rho(l(x-y)), \rho(l(x-T x)), \rho(l(y-T y)))
$$

holds for all $(x, y) \in E(\tilde{G})$ and some $\alpha \in A$, then $T$ is a PO.

Now, we introduce Hardy-Rogers type $(G)_{\rho}$-contraction and obtain related fixed point results.

Definition 3.9 Let $X_{\rho}$ be a modular space. A mapping $T: X_{\rho} \rightarrow X_{\rho}$ is called a HardyRogers type $(G)_{\rho}$-contraction if the following conditions hold:

$\left(\mathrm{H}_{1}\right) T$ preserves edges of $G$;

$\left(\mathrm{H}_{2}\right)$ there exist nonnegative numbers $l_{i}, c$ with $l_{i}<c$ for $i=1, \ldots, 5$ such that

$$
\begin{aligned}
\int_{0}^{\rho(c(T x-T y))} \varphi(t) d t \leq & \eta \int_{0}^{\rho\left(l_{1}(x-y)\right)} \varphi(t) d t+\beta \int_{0}^{\left[\rho\left(l_{2}(x-T x)\right)+\rho\left(l_{3}(y-T y)\right)\right]} \varphi(t) d t \\
& +\gamma \int_{0}^{\left[\rho\left(\frac{l_{4}}{2}(x-T y)\right)+\rho\left(\frac{l_{5}}{2}(y-T x)\right)\right]} \varphi(t) d t
\end{aligned}
$$

holds for each $(x, y) \in E(G)$ with nonnegative numbers $\eta, \beta$, $\gamma$ such that $\eta+2 \beta+2 \gamma<1$ and $\varphi \in \Phi$.

Remark 3.10 Let $X_{\rho}$ be a modular space endowed with a graph $G$ and let $T: X_{\rho} \rightarrow X_{\rho}$ be a Hardy-Rogers type $(G)_{\rho}$-contraction. If there exists $x_{0} \in X_{\rho}$ such that $T x_{0} \in\left[x_{0}\right]_{\tilde{G}}$, then

(i) $T$ is both a Hardy-Rogers type $\left(G^{-1}\right)_{\rho}$-contraction and a Hardy-Rogers type $(\tilde{G})_{\rho}$-contraction,

(ii) $\left[x_{0}\right]_{\tilde{G}}$ is $T$-invariant and $\left.T\right|_{\left[x_{0}\right] \tilde{G}}$ is a Hardy-Rogers type $\left(\tilde{G}_{x_{0}}\right)_{\rho}$-contraction. 
Theorem 3.11 Let $X_{\rho}$ be a $\rho$-complete modular space endowed with a graph $G$, where $\rho$ satisfies the $\Delta_{2}$-condition and let $T: X_{\rho} \rightarrow X_{\rho}$ be a Hardy-Rogers type $(\tilde{G})_{\rho}$-contraction. Assume that the set $X_{T}$ is nonempty and the $C_{\rho}$-graph $G$ is weakly connected. Then $T$ is a PO.

Proof If $x \in X_{T}$, then $T x \in[x]_{\tilde{G}}$ and $\left(T^{n} x, T^{n+1} x\right) \in E(G)$ for all $n \in \mathbb{N}$. Note that

$$
\begin{aligned}
& \int_{0}^{\rho\left(c\left(T^{n} x-T^{n+1} x\right)\right)} \varphi(t) d t \\
& \quad \leq \eta \int_{0}^{\rho\left(l_{1}\left(T^{n-1} x-T^{n} x\right)\right)} \varphi(t) d t+\beta \int_{0}^{\left[\rho\left(l_{2}\left(T^{n-1} x-T^{n} x\right)\right)+\rho\left(l_{3}\left(T^{n} x-T^{n+1} x\right)\right)\right]} \varphi(t) d t \\
& \quad+\gamma \int_{0}^{\left[\rho\left(\frac{l_{4}}{2}\left(T^{n-1} x-T^{n+1} x\right)\right)+\rho\left(\frac{l_{5}}{2}\left(T^{n} x-T^{n} x\right)\right)\right]} \varphi(t) d t \\
& \quad \\
& \quad \eta \int_{0}^{\rho\left(c\left(T^{n-1} x-T^{n} x\right)\right)} \varphi(t) d t+\beta \int_{0}^{\left[\rho\left(c\left(T^{n-1} x-T^{n} x\right)\right)+\rho\left(c\left(T^{n} x-T^{n+1} x\right)\right)\right]} \varphi(t) d t \\
& \quad+\gamma \int_{0}^{\left[\rho\left(c\left(T^{n-1} x-T^{n} x\right)\right)+\rho\left(c\left(T^{n} x-T^{n+1} x\right)\right)\right]} \varphi(t) d t .
\end{aligned}
$$

It follows that

$$
\int_{0}^{\rho\left(c\left(T^{n} x-T^{n+1} x\right)\right)} \varphi(t) d t \leq h \int_{0}^{\rho\left(c\left(T^{n-1} x-T^{n} x\right)\right)} \varphi(t) d t
$$

where $h=\frac{\eta+\beta+\gamma}{1-\beta-\gamma}<1$. Also,

$$
\int_{0}^{\rho\left(c\left(T^{n} x-T^{n+1} x\right)\right)} \varphi(t) d t \leq h^{n} \int_{0}^{\rho(c(x-T x))} \varphi(t) d t .
$$

Taking the limit as $n \rightarrow \infty$, and using Lemma 2.2, we get

$$
\lim _{n} \int_{0}^{\rho\left(c\left(T^{n} x-T^{n+1} x\right)\right)} \varphi(t) d t=0
$$

which implies that $\lim _{n} \rho\left(c\left(T^{n} x-T^{n+1} x\right)\right)=0$.

Let $m, n \in \mathbb{N}$ with $m>n$. By (4) and Remark 2.5, we get

$$
\begin{aligned}
\int_{0}^{\rho\left(\frac{c}{m-n}\left(T^{n} x-T^{m} x\right)\right)} \varphi(t) d t \leq & \int_{0}^{\rho\left(c\left(T^{n} x-T^{n+1} x\right)\right)} \varphi(t) d t+\int_{0}^{\rho\left(c\left(T^{n+1} x-T^{n+2} x\right)\right)} \varphi(t) d t \\
& +\cdots+\int_{0}^{\rho\left(c\left(T^{m-1} x-T^{m} x\right)\right)} \varphi(t) d t \\
\leq & h^{n} \int_{0}^{\rho(c(x-T x))} \varphi(t) d t+h^{n+1} \int_{0}^{\rho(c(x-T x))} \varphi(t) d t \\
& +\cdots+h^{m-1} \int_{0}^{\rho(c(x-T x))} \varphi(t) d t \\
\leq & \frac{h^{n}}{1-h} \int_{0}^{\rho(c(x-T x))} \varphi(t) d t \rightarrow 0, \quad \text { as } n \rightarrow \infty .
\end{aligned}
$$


Thus, $\left\{\frac{c}{m-n} T^{n} x\right\}$ is a $\rho$-Cauchy sequence in $X_{\rho}$. Since $X_{\rho}$ is $\rho$-complete, there exists a point $x^{*} \in X_{\rho}$ such that $\rho\left(\frac{c}{m-n}\left(T^{n} x-x^{*}\right)\right) \rightarrow 0$. As $G$ is a $C_{\rho}$-graph, there exists a subsequence $\left\{T^{n_{p}} x\right\}$ such that $\left(T^{n_{p}} x, x^{*}\right) \in E(G)$ for all $p \in \mathbb{N}$. Also, $\left(T^{n_{p}} x, x^{*}\right) \in E(G)$ for all $p \in \mathbb{N}$. Now we have

$$
\begin{aligned}
\int_{0}^{\rho\left(c\left(T^{n_{p}+1} x-T x^{*}\right)\right)} \varphi(t) d t \leq & \eta \int_{0}^{\rho\left(l_{1}\left(T^{n_{p}} x-x^{*}\right)\right)} \varphi(t) d t \\
& +\beta \int_{0}^{\left[\rho\left(l_{2}\left(T^{n_{p}} x-T^{n_{p}+1} x\right)\right)+\rho\left(l_{3}\left(x^{*}-T x^{*}\right)\right)\right]} \varphi(t) d t \\
& +\gamma \int_{0}^{\left[\rho\left(\frac{l_{4}}{2}\left(T^{n_{p}} x-T x^{*}\right)\right)+\rho\left(\frac{l_{5}}{2}\left(x^{*}-T^{n_{p}+1} x\right)\right)\right]} \varphi(t) d t .
\end{aligned}
$$

Taking the limit as $n \rightarrow \infty$, we have

$$
\int_{0}^{\rho\left(c\left(x^{*}-T x^{*}\right)\right)} \varphi(t) d t \leq(\beta+\gamma) \int_{0}^{\rho\left(c\left(x^{*}-T x^{*}\right)\right)} \varphi(t) d t .
$$

As $(\beta+\gamma)<1$, so $\rho\left(c\left(x^{*}-T x^{*}\right)\right)=0$ and $x^{*}=T x^{*}$.

Next, we prove that $x^{*}$ is a unique fixed point. Suppose that $T$ has another fixed point $y^{*} \in X_{\rho}-\left\{x^{*}\right\}$. Since $G$ is a $C_{\rho}$-graph, there exists a subsequence $\left\{T^{n_{p}} x\right\}$ of $\left\{T^{n} x\right\}$ such that $\left(T^{n_{p}} x, x^{*}\right) \in E(G)$ and $\left(T^{n_{p}} x, y^{*}\right) \in E(G)$ for each $p \in \mathbb{N}$. As $G$ is weakly connected, we have $\left(x^{*}, y^{*}\right) \in E(\tilde{G})$, and

$$
\begin{aligned}
\int_{0}^{\rho\left(c\left(x^{*}-y^{*}\right)\right)} \varphi(t) d t & =\int_{0}^{\rho\left(c\left(T x^{*}-T y^{*}\right)\right)} \varphi(t) d t \\
\leq & \eta \int_{0}^{\rho\left(l_{1}\left(x^{*}-y^{*}\right)\right)} \varphi(t) d t+\beta \int_{0}^{\left[\rho\left(l_{2}\left(x^{*}-T x^{*}\right)\right)+\rho\left(l_{3}\left(y^{*}-T y^{*}\right)\right)\right]} \varphi(t) d t \\
& +\gamma \int_{0}^{\left[\rho\left(\frac{l_{4}}{2}\left(x^{*}-T y^{*}\right)\right)+\rho\left(\frac{l_{5}}{2}\left(y^{*}-T x^{*}\right)\right)\right]} \varphi(t) d t
\end{aligned}
$$

which further implies that

$$
\int_{0}^{\rho\left(c\left(x^{*}-y^{*}\right)\right)} \varphi(t) d t \leq(\eta+2 \gamma) \int_{0}^{\rho\left(c\left(x^{*}-y^{*}\right)\right)} \varphi(t) d t .
$$

Since $(\eta+2 \gamma)<1, \int_{0}^{\rho\left(c\left(x^{*}-y^{*}\right)\right)} \varphi(t) d t=0$. The result follows.

In Theorem 3.11, if we replace the condition that $G$ is a $C_{\rho}$-graph with orbitally $G_{\rho}$ continuity of $T$, then we have the following theorem.

Theorem 3.12 Let $X_{\rho}$ be a $\rho$-complete modular space endowed with a graph $G$, where $\rho$ satisfies the $\Delta_{2}$-condition and let $T: X_{\rho} \rightarrow X_{\rho}$ be a Hardy-Rogers type $(\tilde{G})_{\rho}$-contraction, which is orbitally $G_{\rho}$-continuous. Assume that the set $X_{T}$ is nonempty and the graph $G$ is weakly connected. Then $T$ is a PO.

In the following suppose that $X_{\rho}$ is a $\rho$-complete modular space endowed with a graph $G$, where $\rho$ satisfies the $\Delta_{2}$-condition and $T: X_{\rho} \rightarrow X_{\rho}$ is edge-preserving such that the set $X_{T}$ is nonempty. 


\section{Corollary 3.13 Assume}

(i) the $C_{\rho}$-graph $G$ is weakly connected and

(ii) there exist nonnegative numbers $l, c$ with $l<c$ such that

$$
\int_{0}^{\rho(c(T x-T y))} \varphi(t) d t \leq \eta \int_{0}^{\rho(l(x-y))} \varphi(t) d t
$$

holds for each $(x, y) \in E(\tilde{G})$ with $\eta \in(0,1)$ and $\varphi \in \Phi$. Then $T$ is a PO.

\section{Corollary 3.14 Assume}

(i) the $C_{\rho}$-graph $G$ is weakly connected and

(ii) there exist nonnegative numbers $l_{1}, l_{2}, c$ with $l_{1}, l_{2}<c$ such that

$$
\int_{0}^{\rho(c(T x-T y))} \varphi(t) d t \leq \beta \int_{0}^{\left[\rho\left(l_{1}(x-T x)\right)+\rho\left(l_{2}(y-T y)\right)\right]} \varphi(t) d t
$$

holds for each $(x, y) \in E(\tilde{G})$ with $\beta \in\left(0, \frac{1}{2}\right)$ and $\varphi \in \Phi$. Then $T$ is a PO.

\section{Corollary 3.15 Assume}

(i) the $C_{\rho}$-graph $G$ is weakly connected and

(ii) there exist nonnegative numbers $l_{1}, l_{2}, c$ with $l_{1}, l_{2}<c$ such that

$$
\int_{0}^{\rho(c(T x-T y))} \varphi(t) d t \leq \gamma \int_{0}^{\left[\rho\left(\frac{l_{1}}{2}(x-T y)\right)+\rho\left(\frac{l_{2}}{2}(y-T x)\right)\right]} \varphi(t) d t
$$

for each $(x, y) \in E(\tilde{G})$ with $\gamma \in\left(0, \frac{1}{2}\right)$ and $\varphi \in \Phi$. Then $T$ is a PO.

Now we introduce the $(G, \phi, \psi)_{\rho}$-contraction and obtain some fixed point results.

Definition 3.16 A mapping $T: X_{\rho} \rightarrow X_{\rho}$ is called a $(G, \phi, \psi)_{\rho}$-contraction if the following conditions hold:

$\left(\mathrm{Q}_{1}\right) T$ preserves edges of $G$;

$\left(\mathrm{Q}_{2}\right)$ there exist nonnegative numbers $l, c$ with $l<c$ such that

$$
\int_{0}^{\psi(\rho(c(T x-T y)))} \varphi(t) d t \leq \phi\left(\int_{0}^{\psi(\rho(l(x-y)))} \varphi(t) d t\right)
$$

holds for each $(x, y) \in E(G)$, where $\psi \in \Psi, \phi \in \Phi_{1}$, and $\varphi \in \Phi$.

Remark 3.17 Let $T: X_{\rho} \rightarrow X_{\rho}$ be a $(G, \phi, \psi)_{\rho}$-contraction. If there exists $x_{0} \in X_{\rho}$ such that $T x_{0} \in\left[x_{0}\right]_{\tilde{G}}$, then

(i) $T$ is both a $\left(G^{-1}, \phi, \psi\right)_{\rho}$-contraction and a $(\tilde{G}, \phi, \psi)_{\rho}$-contraction,

(ii) $\left[x_{0}\right]_{\tilde{G}}$ is $T$-invariant and $\left.T\right|_{\left[x_{0}\right] \tilde{G}}$ is a $\left(\tilde{G}_{x_{0}}, \phi, \psi\right)_{\rho}$-contraction.

Theorem 3.18 Let $X_{\rho}$ be a $\rho$-complete modular space endowed with a graph $G$, where $\rho$ satisfies the $\Delta_{2}$-condition and let $T: X_{\rho} \rightarrow X_{\rho}$ be a $(\tilde{G}, \phi, \psi)_{\rho}$-contraction. If $X_{T}$ is nonempty and $C_{\rho}$-graph $G$ is weakly connected, then $T$ is a PO. 
Proof If $x \in X_{T}$, then $\left(T^{n} x, T^{n+1} x\right) \in E(G)$ for all $n \in \mathbb{N}$. First, we show that the sequence $\left\{\psi\left(\rho\left(c\left(T^{n} x-T^{n+1} x\right)\right)\right)\right\}$ converges to 0 . From Definition 3.16, we have

$$
\begin{aligned}
\int_{0}^{\psi\left(\rho\left(c\left(T^{n} x-T^{n+1} x\right)\right)\right)} \varphi(t) d t & \leq \phi\left(\int_{0}^{\psi\left(\rho\left(l\left(T^{n-1} x-T^{n} x\right)\right)\right)} \varphi(t) d t\right) \\
& <\int_{0}^{\psi\left(\rho\left(l\left(T^{n-1} x-T^{n} x\right)\right)\right)} \varphi(t) d t \\
& <\int_{0}^{\psi\left(\rho\left(c\left(T^{n-1} x-T^{n} x\right)\right)\right)} \varphi(t) d t .
\end{aligned}
$$

Thus,

$$
\left\{\int_{0}^{\psi\left(\rho\left(c\left(T^{n} x-T^{n+1} x\right)\right)\right)} \varphi(t) d t\right\}
$$

is decreasing and bounded from below and so

$$
\left\{\int_{0}^{\psi\left(\rho\left(c\left(T^{n} x-T^{n+1} x\right)\right)\right)} \varphi(t) d t\right\}
$$

converges to a nonnegative number $L$. If $L \neq 0$, we obtain

$$
\begin{aligned}
L & =\lim _{n \rightarrow \infty} \int_{0}^{\psi\left(\rho\left(c\left(T^{n} x-T^{n+1} x\right)\right)\right)} \varphi(t) d t \leq \lim _{n \rightarrow \infty} \phi\left(\int_{0}^{\psi\left(\rho\left(l\left(T^{n-1} x-T^{n} x\right)\right)\right)} \varphi(t) d t\right) \\
& \leq \lim _{n \rightarrow \infty} \phi\left(\int_{0}^{\psi\left(\rho\left(c\left(T^{n-1} x-T^{n} x\right)\right)\right)} \varphi(t) d t\right),
\end{aligned}
$$

that is, $L \leq \phi(L)$, a contradiction. Hence $\int_{0}^{\psi\left(\rho\left(c\left(T^{n} x-T^{n+1} x\right)\right)\right)} \varphi(t) d t \rightarrow 0$ as $n \rightarrow \infty$. It follows that $\psi\left(\rho\left(c\left(T^{n} x-T^{n+1} x\right)\right)\right) \rightarrow 0$. Suppose that

$$
\lim _{n \rightarrow \infty} \sup \psi\left(\rho\left(c\left(T^{n} x-T^{n+1} x\right)\right)\right)=\varepsilon>0 .
$$

Then there exists a $v_{\varepsilon} \in \mathbb{N}$ and a sequence $\left\{T^{n_{\nu}} x\right\}_{v \geq v_{\varepsilon}}$ such that

$$
\begin{aligned}
& \psi\left(\rho\left(c\left(T^{n_{v}} x-T^{n_{v}+1} x\right)\right)\right) \rightarrow \varepsilon>0, \quad v \rightarrow \infty, \\
& \psi\left(\rho\left(c\left(T^{n_{v}} x-T^{n_{v}+1} x\right)\right)\right) \geq \frac{\varepsilon}{2}, \quad \forall v \geq v_{\varepsilon} .
\end{aligned}
$$

Hence we get the following:

$$
0=\lim _{\nu \rightarrow \infty} \int_{0}^{\psi\left(\rho\left(c\left(T^{n_{\nu}} x-T^{n_{\nu}+1} x\right)\right)\right)} \varphi(t) d t \geq \int_{0}^{\frac{\varepsilon}{2}} \varphi(t) d t>0 .
$$

Assume that there is an $\varepsilon>0$ and there exist $m_{v}, n_{v} \in \mathbb{N}$ such that $m_{v}>n_{v}>v$ for each $v \in \mathbb{N}$ and

$$
\psi\left(\rho\left(l\left(T^{m_{v}} x-T^{n_{v}} x\right)\right)\right) \geq \varepsilon .
$$


Then we choose the sequence $\left(m_{v}\right)_{v \in \mathbb{N}}$ and $\left(n_{v}\right)_{v \in \mathbb{N}}$ such that for each $v \in \mathbb{N}, m_{v}$ is minimal in the sense that $\psi\left(\rho\left(l\left(T^{m_{v}} x-T^{n_{v}} x\right)\right)\right) \geq \varepsilon$ but $\psi\left(\rho\left(l\left(T^{s} x-T^{n_{v}} x\right)\right)\right)<\varepsilon$, for all $s \in$ $\left\{n_{v}+1, \ldots, m_{v}-1\right\}$. Now, let $\delta \in \mathbb{R}_{+}$be such that $\frac{l}{c}+\frac{1}{\delta}=1$, then we have

$$
\begin{aligned}
& \int_{0}^{\varepsilon} \varphi(t) d t \leq \int_{0}^{\psi\left(\rho\left(l\left(T^{m_{v}} x-T^{n_{\nu}} x\right)\right)\right)} \varphi(t) d t \\
& \leq \int_{0}^{\psi\left(\rho\left(c\left(T^{m_{\nu}} x-T^{n_{\nu}+1} x\right)\right)\right)} \varphi(t) d t+\int_{0}^{\psi\left(\rho\left(\delta l\left(T^{n_{\nu}+1} x-T^{n_{\nu}} x\right)\right)\right)} \varphi(t) d t \\
& \leq \phi\left(\int_{0}^{\psi\left(\rho\left(l\left(T^{m_{\nu}-1} x-T^{n_{\mathcal{V}}} x\right)\right)\right)} \varphi(t) d t\right)+\int_{0}^{\psi\left(\rho\left(\delta l\left(T^{n_{\mathcal{V}}+1} x-T^{n_{\mathcal{V}}} x\right)\right)\right)} \varphi(t) d t \\
& \leq \int_{0}^{\psi\left(\rho\left(l\left(T^{m_{\nu}-1} x-T^{n_{\nu}} x\right)\right)\right)} \varphi(t) d t+\int_{0}^{\psi\left(\rho\left(\delta l\left(T^{n_{\nu}+1} x-T^{n_{\nu}} x\right)\right)\right)} \varphi(t) d t \\
& \leq \int_{0}^{\varepsilon} \varphi(t) d t+\int_{0}^{\psi\left(\rho\left(\delta l\left(T^{n_{\nu}+1} x-T^{n_{\nu}} x\right)\right)\right)} \varphi(t) d t .
\end{aligned}
$$

Thus, taking the limit as $v \rightarrow \infty$, and Proposition 2.8, we have

$$
\int_{0}^{\psi\left(\rho\left(\delta l\left(T^{n_{v}+1} x-T^{n_{\nu}} x\right)\right)\right)} \varphi(t) d t \rightarrow 0
$$

Therefore,

$$
\int_{0}^{\psi\left(\rho\left(l\left(T^{m_{v}} x-T^{n_{v}} x\right)\right)\right)} \varphi(t) d t \rightarrow \varepsilon, \quad v \rightarrow \infty
$$

Now,

$$
\begin{aligned}
& \int_{0}^{\psi\left(\rho\left(l\left(T^{m_{\nu}} x-T^{n_{\nu}} x\right)\right)\right)} \varphi(t) d t \\
& \leq \int_{0}^{\psi\left(\rho\left(c\left(T^{m_{\nu}+1} x-T^{n_{\nu}+1} x\right)\right)\right)} \varphi(t) d t+\int_{0}^{\psi\left(\rho\left(2 \delta l\left(T^{n_{\nu}+1} x-T^{n_{\nu}} x\right)\right)\right)} \varphi(t) d t \\
& \quad+\int_{0}^{\psi\left(\rho\left(2 \delta l\left(T^{m_{\nu}+1} x-T^{m_{\nu}} x\right)\right)\right)} \varphi(t) d t \\
& \leq \phi\left(\int_{0}^{\psi\left(\rho\left(l\left(T^{m_{\nu}} x-T^{n_{\nu}} x\right)\right)\right)} \varphi(t) d t\right)+\int_{0}^{\psi\left(\rho\left(2 \delta l\left(T^{n_{\nu}+1} x-T^{n_{\nu}} x\right)\right)\right)} \varphi(t) d t \\
& \quad+\int_{0}^{\psi\left(\rho\left(2 \delta l\left(T^{m_{\nu}+1} x-T^{m_{\nu}} x\right)\right)\right)} \varphi(t) d t .
\end{aligned}
$$

If $v \rightarrow \infty$, then we have

$$
\int_{0}^{\varepsilon} \varphi(t) d t \leq \phi\left(\int_{0}^{\varepsilon} \varphi(t) d t\right)
$$

a contradiction for $\varepsilon>0$. Hence, $\left\{l T^{n} x\right\}$ is a $\rho$-Cauchy sequence. By $\rho$-completeness of $X_{\rho}$, there exists $x^{*} \in X_{\rho}$ such that $\rho\left(l\left(T^{n} x-x^{*}\right)\right) \rightarrow 0$ as $n \rightarrow \infty$ and $\left(T^{n} x, T^{n+1} x\right) \in E(G)$ for all $n \in \mathbb{N}$ and $G$ is a $C_{\rho}$-graph, then there exists a subsequence $\left\{T^{n_{p}} x\right\}$ such that $\left(T^{n_{p}} x, x^{*}\right) \in$ 
$E(G)$ for each $p \in \mathbb{N}$. Also, $\left(T^{n_{p}} x, x^{*}\right) \in E(G)$ for each $p \in \mathbb{N}$. From Remark 2.5 and $\left(\psi_{3}\right)$, it follows that

$$
\begin{aligned}
\psi\left(\rho\left(\frac{c}{2}\left(x^{*}-T x^{*}\right)\right)\right) & =\psi\left(\rho\left(\frac{c}{2}\left(x^{*}-T^{n_{p}+1} x\right)+\frac{c}{2}\left(T^{n_{p}+1} x-T x^{*}\right)\right)\right) \\
& \leq \psi\left(\rho\left(c\left(x^{*}-T^{n_{p}+1} x\right)\right)+\rho\left(c\left(T^{n_{p}+1} x-T x^{*}\right)\right)\right) \\
& \leq \psi\left(\rho\left(c\left(x^{*}-T^{n_{p}+1} x\right)\right)\right)+\psi\left(\rho\left(c\left(T^{n_{p}+1} x-T x^{*}\right)\right)\right) .
\end{aligned}
$$

Taking the limit as $p \rightarrow \infty$, we have

$$
\begin{aligned}
\int_{0}^{\psi\left(\rho\left(c\left(T^{n_{p}+1} x-T x^{*}\right)\right)\right)} \varphi(t) d t & \leq \phi\left(\int_{0}^{\psi\left(\rho\left(l\left(T^{n_{p}} x-x^{*}\right)\right)\right)} \varphi(t) d t\right) \\
& \leq \int_{0}^{\psi\left(\rho\left(l\left(T^{n_{p}} x-x^{*}\right)\right)\right)} \varphi(t) d t .
\end{aligned}
$$

Since $\rho\left(l\left(T^{n_{p}} x-x^{*}\right)\right) \rightarrow 0(p \rightarrow \infty)$, we obtain

$$
\lim _{p \rightarrow \infty} \int_{0}^{\psi\left(\rho\left(l\left(T^{n_{p}} x-x^{*}\right)\right)\right)} \varphi(t) d t \leq 0
$$

which implies that $\psi\left(\rho\left(c\left(T^{n_{p}+1} x-T x^{*}\right)\right)\right) \rightarrow 0$, as $p \rightarrow \infty$. Thus,

$$
\psi\left(\rho\left(c\left(x^{*}-T^{n_{p}+1} x\right)\right)\right)+\psi\left(\rho\left(c\left(T^{n_{p}+1} x-T x^{*}\right)\right)\right) \rightarrow 0, \quad \text { as } p \rightarrow \infty .
$$

Hence $\lim _{n \rightarrow \infty} \psi\left(\rho\left(\frac{c}{2}\left(x^{*}-T x^{*}\right)\right)\right)=0$ and $x^{*}=T x^{*}$.

Finally, we prove that $x^{*}$ is a unique fixed point. Suppose that $T$ has another fixed point $y^{*} \in X_{\rho}-\left\{x^{*}\right\}$. Since $G$ is a $C_{\rho}$-graph, there exists a subsequence $\left\{T^{n_{p}} x\right\}$ of $\left\{T^{n} x\right\}$ such that $\left(T^{n_{p}} x, x^{*}\right) \in E(G)$ and $\left(T^{n_{p}} x, y^{*}\right) \in E(G)$ for each $p \in \mathbb{N}$. Furthermore, as $G$ is weakly connected, $\left(x^{*}, y^{*}\right) \in E(\tilde{G})$. We have

$$
\begin{aligned}
\int_{0}^{\psi\left(\rho\left(c\left(x^{*}-y^{*}\right)\right)\right)} \varphi(t) d t & =\int_{0}^{\psi\left(\rho\left(c\left(T x^{*}-T y^{*}\right)\right)\right)} \varphi(t) d t \\
& \leq \phi\left(\int_{0}^{\psi\left(\rho\left(l\left(x^{*}-y^{*}\right)\right)\right)} \varphi(t) d t\right) \\
& <\int_{0}^{\psi\left(\rho\left(l\left(x^{*}-y^{*}\right)\right)\right)} \varphi(t) d t \\
& <\int_{0}^{\psi\left(\rho\left(c\left(x^{*}-y^{*}\right)\right)\right)} \varphi(t) d t,
\end{aligned}
$$

a contradiction. Hence, $x^{*}=y^{*}$.

In Theorem 3.18, if we replace the condition that $G$ is a $C_{\rho}$-graph with orbitally $G_{\rho^{-}}$ continuity of $T$, then we have the following theorem.

Theorem 3.19 Let $X_{\rho}$ be a $\rho$-complete modular space endowed with a graph $G$, where $\rho$ satisfies the $\Delta_{2}$-condition and let $T: X_{\rho} \rightarrow X_{\rho}$ be a $(\tilde{G}, \phi, \psi)_{\rho}$-contraction, which is orbitally $G_{\rho}$-continuous. Assume that the set $X_{T}$ is nonempty and the graph $G$ is weakly connected. Then $T$ is a PO. 
In the following corollaries, suppose that $X_{\rho}$ is a $\rho$-complete modular space endowed with a graph $G$, where $\rho$ satisfies the $\Delta_{2}$-condition and let $T: X_{\rho} \rightarrow X_{\rho}$ be edge-preserving and the set $X_{T}$ be nonempty.

\section{Corollary 3.20 Assume}

(i) the $C_{\rho}$-graph $G$ is weakly connected and

(ii) there exist nonnegative numbers $l, c$ with $l<c$ such that

$$
\int_{0}^{\rho(c(T x-T y))} \varphi(t) d t \leq \phi\left(\int_{0}^{\rho(l(x-y))} \varphi(t) d t\right)
$$

hold for each $(x, y) \in E(\tilde{G})$ with $\phi \in \Phi_{1}$ and $\varphi \in \Phi$. Then $T$ is a PO.

\section{Corollary 3.21 Assume}

(i) the $C_{\rho}$-graph $G$ is weakly connected and

(ii) there exist nonnegative numbers $l, c$ with $l<c$ such that

$$
\int_{0}^{\psi(\rho(c(T x-T y)))} \varphi(t) d t \leq \eta \int_{0}^{\psi(\rho(l(x-y)))} \varphi(t) d t
$$

for each $(x, y) \in E(\tilde{G})$ with $\eta \in(0,1)$ and $\varphi \in \Phi$. Then $T$ is a PO.

Now we provide examples in support of our results.

Example 3.22 Let $X_{\rho}=\{0,1,2,3,4,5\}$ and $\rho(x)=|x|$, for all $x \in X_{\rho}$. Consider

$$
E(\tilde{G})=\{(1,1),(2,2),(3,3),(4,4),(5,5)\} \cup\left\{(0, x): x \in X_{\rho}\right\},
$$

and $T x=0, x \in X_{\rho}$. Then $G$ is weakly connected and $C_{\rho}$-graph, $X_{T}$ is nonempty and $T$ is a $(\tilde{G}, A)_{\rho}$-contraction where $c=\frac{7}{5}, l=\frac{6}{5}, \varphi(t)=1$. Hence, $T$ is a PO.

Example 3.23 Let $X_{\rho}=\{0,1,2,3\}$ and $\rho(x)=|x|$, for all $x \in X_{\rho}$. Consider

$$
E(\tilde{G})=\{(0,0),(0,1),(1,1),(1,3),(2,2),(0,3),(2,3),(3,3)\}
$$

Define $T: X_{\rho} \rightarrow X_{\rho}$ as follows:

$$
T x= \begin{cases}0, & x \in\{0,1\} \\ 1, & x \in\{2,3\}\end{cases}
$$

Then $G$ is weakly connected and $C_{\rho}$-graph, $X_{T}$ is nonempty, and $T$ is a Hardy-Rogers type $(\tilde{G})_{\rho}$-contraction where $c=4, l_{1}=l_{2}=l_{3}=3, l_{4}=l_{5}=\frac{1}{2}, \eta=\frac{1}{3}, \beta=\frac{1}{4}, \gamma=0$, and $\varphi(t)=1$. Moreover, 0 is a unique fixed point of $T$.

Example 3.24 Let $X_{\rho}=[0,1]$ and $\rho(x)=|x|$, for all $x \in X_{\rho}$. Consider

$$
E(G)=\{(0,0)\} \cup\{(0, x): x \geq 1 / 2\} \cup\{(x, y): x, y \in(0,1]\},
$$


and $T x=\frac{x}{2}, x \in X_{\rho}$. Then $G$ is weakly connected and a $C_{\rho}$-graph, $X_{T}$ is nonempty and $T$ is a $(\tilde{G}, \phi, \psi)_{\rho}$-contraction where $c=\frac{1}{2}, l=\frac{1}{3}, \varphi(t)=1, \phi(\xi)=\frac{\xi}{1+\xi}$, and $\psi(\omega)=\frac{\omega}{3}$. Thus all conditions of Theorem 3.18 are satisfied. Moreover, $T$ is a PO.

Remark 3.25 In the above examples, if we use $\rho(x)=x^{2}$, the conclusions remain the same.

\section{Competing interests}

The authors declare that they have no competing interests.

\section{Authors' contributions}

All authors contributed equally to the writing of this paper. All authors read and approved the final manuscript.

\section{Author details}

'Department of Mathematics, Sakarya University, Sakarya, 54187, Turkey. ${ }^{2}$ Department of Mathematics and Applied Mathematics, University of Pretoria, Lynnwood Road, Pretoria, 0002, South Africa. ${ }^{3}$ Department of Mathematics, Lahore University of Management Sciences (LUMS), Phase-II, Opposite sector U, DHA Lahore Cantt., Lahore, 54792, Pakistan.

\section{Acknowledgements}

The authors are thankful to the anonymous referees for their valuable comments and suggestions, which helped to improve the presentation of this paper.

\section{Received: 30 May 2014 Accepted: 14 October 2014 Published: 29 Oct 2014}

\section{References}

1. Banach, S: Sur les opérations dans les ensembles abstraits et leur application aux équations intégrales. Fundam. Math. 3, 133-181 (1922)

2. Ran, ACM, Reuring, MCB: A fixed point theorem in partially ordered sets and some applications to matrix equations. Proc. Am. Math. Soc. 132, 1435-1443 (2004)

3. Abbas, M, Khamsi, MA, Khan, AR: Common fixed point and invariant approximation in hyperbolic ordered metric spaces. Fixed Point Theory Appl. 2011, Article ID 25 (2011). doi:10.1186/1687-1812-2011-25

4. Ćirić, L, Abbas, M, Saadati, R, Hussain, N: Common fixed points of almost generalized contractive mappings in ordered metric spaces. Appl. Math. Comput. 217, 5784-5789 (2011)

5. Jachymski, J: The contraction principle for mappings on a metric space endowed with a graph. Proc. Am. Math. Soc. $136,1359-1373(2008)$

6. Abbas, M, Nazir, T: Common fixed point of a power graphic contraction pair in partial metric spaces endowed with a graph. Fixed Point Theory Appl. 2013, Article ID 20 (2013)

7. Öztürk, M, Girgin, E: On some fixed-point theorems for $\psi$-contraction on metric space involving a graph. J. Inequal. Appl. 2014, Article ID 39 (2014). doi:10.1186/1029-242X-2014-39

8. Samreen, M, Kamran, T: Fixed point theorems for integral G-contractions. Fixed Point Theory Appl. 2013, Article ID 149 (2013)

9. Kannan, R: Some results on fixed points. Bull. Calcutta Math. Soc. 60, 71-76 (1968)

10. Rhaodes, BE: A comparison of various definitions of contractive mappings. Trans. Am. Math. Soc. 26, $257-290$ (1977)

11. Branciari, A: A fixed point theorem for mappings satisfying a general contractive condition of integral type. Int. J. Math. Math. Sci. 29, 531-536 (2002)

12. Akram, M, Zafar, AA, Siddiqui, AA: A general class of contractions: A-contractions. Novi Sad J. Math. 38(1), 25-33 (2002)

13. Bianchini, R: Su un problema di S. Reich riguardante la teoria dei punti fissi. Boll. Unione Mat. Ital. 5, 103-108 (1972)

14. Reich, S: Kannan's fixed point theorem. Boll. Unione Mat. Ital. 5, 1-11 (1971)

15. Nakano, H: Modulared Semi-Ordered Linear Spaces. Tokyo Math. Book Ser., vol. 1. Maruzen, Tokyo (1950)

16. Musielak, T, Orlicz, W: On modular spaces. Stud. Math. 18, 49-65 (1959)

17. Koshi, S, Shimogaki, T: On F-norms of quasi-modular spaces. J. Fac. Sci. Hokkaido Univ., Ser. I 15(3-4), 202-218 (1961)

18. Mazur, S, Orlicz, W: On some classes of linear spaces. Stud. Math. 17, 97-119 (1958) (Reprinted in Wladyslaw Orlicz Collected Papers, pp. 981-1003, PWN, Warszawa (1988))

19. Yamamuro, S: On conjugate spaces of Nakano spaces. Trans. Am. Math. Soc. 90, 291-311 (1959)

20. Turpin, PH: Fubini inequalities and bounded multiplier property in generalized modular spaces. Comment. Math. 1 331-353 (1978) (Tomus specialis in honorem Ladislai Orlicz)

21. Luxemburg, WAJ: Banach function spaces. Thesis, Delft Inst. of Techn. Asser., The Netherlands (1955)

22. Khamsi, MA, Kozolowski, WK, Reich, S: Fixed point theory in modular function spaces. Nonlinear Anal. 14, 935-953 (1990)

23. Benavides, TD, Khamsi, MA, Samadi, S: Asymptotically non-expansive mappings in modular function spaces. J. Math. Anal. Appl. 265, 249-263 (2002)

24. Benavides, TD, Khamsi, MA, Samadi, S: Asymptotically regular mappings in modular function spaces. Sci. Math. Jpn. 53, 295-304 (2001)

25. Benavides, TD, Khamsi, MA, Samadi, S: Uniformly Lipschitzian mappings in modular function spaces. Nonlinear Anal. 46, 267-278 (2001)

26. Khamsi, MA: A convexity property in modular function spaces. Math. Jpn. 44, 269-279 (1996)

27. Mongkolkeha, C, Kumam, P: Fixed point and common fixed point theorems for generalized weak contraction mappings of integral type in modular spaces. Int. J. Math. Math. Sci. 2011, Article ID 705943 (2011) 
28. Beygmohammadi, M, Razani, A: Two fixed-point theorems for mappings satisfying a general contractive condition of integral type in the modular space. Int. J. Math. Math. Sci. 2010, Article ID 317107 (2010)

29. Liu, Z, Li, X, Kang, SM, Cho, SY: Fixed point theorems for mappings satisfying contractive conditions of integral type and applications. Fixed Point Theory Appl. 2011, Article ID 64 (2011)

30. Mongkolkeha, C, Kumam, P: Some fixed point results for generalized weak contraction mappings in modular spaces. Int. J. Anal. 2013, Article ID 247378 (2013)

10.1186/1687-1812-2014-220

Cite this article as: Öztürk et al.: Fixed points of mappings satisfying contractive condition of integral type in modular spaces endowed with a graph. Fixed Point Theory and Applications 2014, 2014:220

Submit your manuscript to a SpringerOpen ${ }^{\circ}$ journal and benefit from:

- Convenient online submission

- Rigorous peer review

- Immediate publication on acceptance

- Open access: articles freely available online

- High visibility within the field

- Retaining the copyright to your article

Submit your next manuscript at $>$ springeropen.com 